\title{
Familial cerebral amyloidosis and spongiform encephalopathy
}

\author{
JANE ADAM, TJ CROW, LW DUCHEN, F SCARAVILLI, E SPOKES* \\ From the Department of Neuropathology, Institute of Neurology, The National Hospital, \\ Queen Square, London and the Clinical Research Centre, Northwick Park, Harrow
}

SUMMARY Clinical and neuropathological investigations are presented of the "W" family in which there is a dominant inheritance of slowly progressive cerebellar ataxia and dementia. The disease is of insidious onset and its average duration more than 4 years. Pathological findings included amyloid deposition in cerebellar and cerebral tissue; vascular amyloid in one case; and spongiform encephalopathy and astrocytic hyperplasia typical of Creutzfeldt-Jakob disease. Neuritic plaques of the senile (Alzheimer) type were present to a lesser extent. This study confirms the familial association between cerebral amyloidosis and spongiform encephalopathy.

Progressive neurological disorders with dementia known to have an hereditary basis include Alzheimer's disease particularly in its atypical forms $^{12}$ and Creutzfeldt-Jakob disease (CJD). ${ }^{3}$ In an analysis of many families with CJD it was estimated ${ }^{4}$ that more than $15 \%$ of cases occurred within families in which two or more members were affected. Since CJD has been transmitted by intracerebral inoculation into susceptible animals ${ }^{5}$ the relationship between heredity and infectivity poses considerable problems.

In this paper we present the clinical histories and neuropathological findings in four members of the "W" family of which 23 members in seven generations (fig 1) were affected by a progressive neurological disease usually with dementia. The family's origins are in Bedfordshire, England and seven generations were traced back to 1791 by Cameron and Crawford, ${ }^{6}$ though no details of the five earliest affected members are available. The disease has affected both males and females and has been transmitted by both males and females. The pattern of inheritance is thus consistent with transmission by an autosomal dominant gene of complete penetrance. The morphologica! features of the brains of these four cases include spongiform encephalopathy,

*Present address: Department of Neurology, General Infirmary, Leeds.

Address for reprint requests: Professor LW Duchen, Department of Neuropathology, Institute of Neurology, The National Hospital, Queen Square, London WCIN 3BG, UK.

Received 31 July 1981

Accepted 3 September 1981 amyloid and astrocytic hyperplasia--characteristics of CJD. One other member (JW) of this family had emigrated to the USA and developed CJD. ${ }^{7}$ Inoculation of cerebral tissue from this case into nonhuman primates transmitted an encephalopathy. ${ }^{4}$

\section{Clinical histories}

Case 1 JM. Male, born 1912. Fifth generation, III-25 in the pedigree of Rosenthal et al. ${ }^{7}$ In 1972 he began to have difficulty in remembering. In 1973 examination showed little abnormality except brisk reflexes with flexor plantar responses and psychometric testing revealed generalised intellectual impairment. The EEG record was described as "poorly organised". Examination of cerebrospinal fluid and haematological investigations gave normal results. Air encephalography showed enlarged ventricles particularly of the frontal horns. Intellectual functions deteriorated and he died in 1975. Postmortem examination showed terminal pneumonia and it was noted that the cardiovascular system was virtually free of atheroma. The brain and spinal cord were received at the National Hospital after fixation.

Case 2 JJ. Female, born 1920. Fifth generation, III-38 in the pedigree of Rosenthal et al. ${ }^{7}$ Until 1970 this patient was well except for a right-sided below-knee amputation for osteomyelitis at age 9. At the age of $50 \mathrm{yr}$ she began to experience difficulty in walking and within 2 years was admitted to hospital unable to get out of bed or walk without assistance. She was severely depressed and withdrawn, forgetful and confused, neglectful of personal hygiene and doubly incontinent. On examination she was demented with slurring of speech, a flaccid hemiparesis and hypotonia of both arms. By 1973 she was debilitated, mute, unresponsive and totally bedridden. Pupils were 


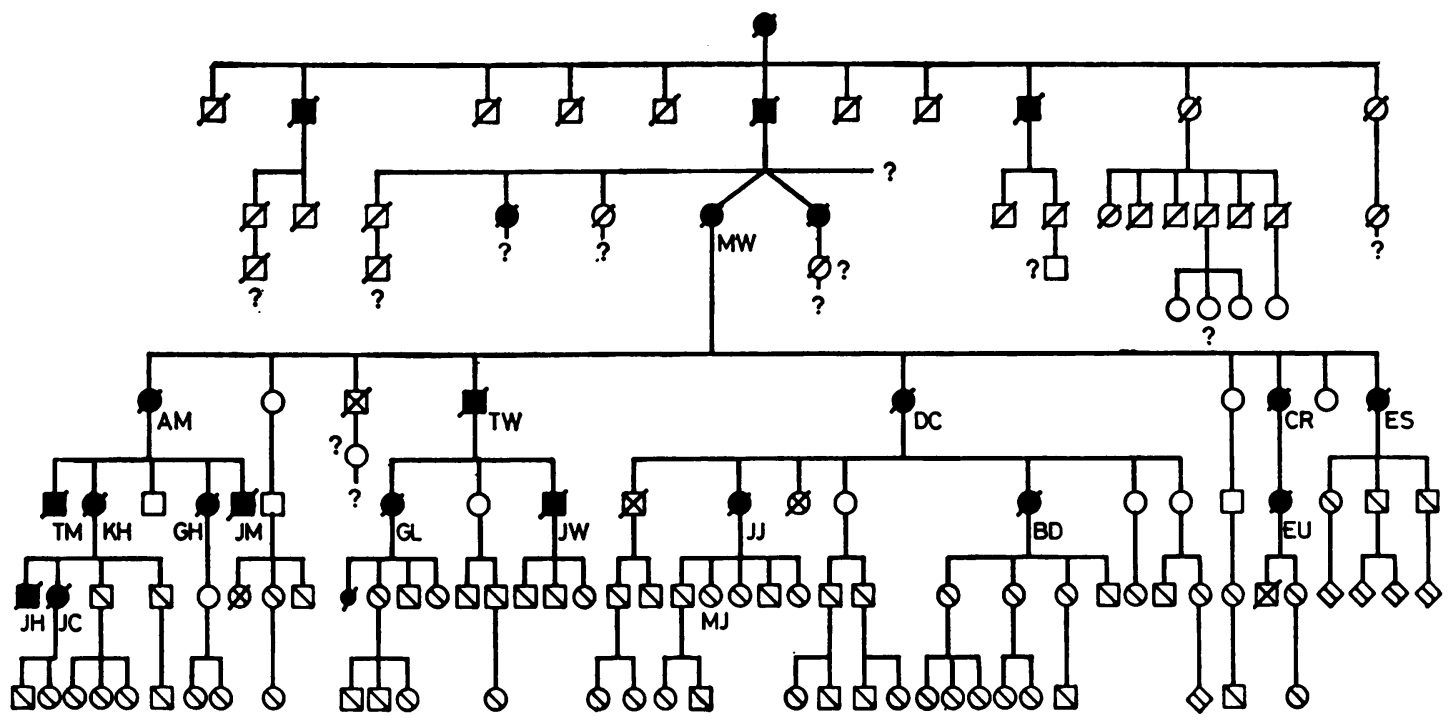

Fig 1 Family tree. $\square$-male; $\bigcirc-$ female; $\bigcirc-$ sex unknown; solid symbol-affected by progressive neurological disease; open symbol-unaffected aged over 50; \-aged under 50; $/$-deceased; $\times$-deceased under 50; ?-cause of death unknown; |?-descendants untraced; $\checkmark$-infant death; only limited information about age of death and descendants in generations I-III and Part of IV.

dilated and reacted sluggishly to light and left disc pallor was noted. There was no nystagmus. She died of bronchopneumonia 3 years after the onset of her illness. The postmortem examination was done at the National Hospital (Professor Blackwood).

Case 3 EU. Female, born 1914. Fifth generation, III50 in the pedigree of Rosenthal et al. ${ }^{7}$ She was well until 1971 when she became unsteady on her feet, had numerous falls and sustained minor injuries. In 1972 she was noted to be depressed but without abnormal physical signs. Within a year her unsteadiness was worse, she had difficulty in climbing stairs, was tearful and slept poorly. Examination showed dysarthria, an immobile face with a positive jaw jerk and a left hemiparesis with a left extensor plantar response. By 1974 she was confined to a wheel chair because of progressive weakness and ataxia and her handwriting was illegible. Her final admission to hospital was in 1975 by which time she was withdrawn, confused, disorientated and doubly incontinent. There was no spontaneous speech and mixed dysphasia was noted. Marked weakness of both lower limbs was present with absent deep tendon reflexes and bilateral extensor plantar responses. Investigations which gave normal results included ESR, urea and electrolytes, liver function tests, B12, folate, chest and skull radiographs and WR. Haemoglobin was $17 \mathrm{~g} / \mathrm{dl}$, white cell count raised at $17000 / \mathrm{mm}^{3}$ with a normal differential, a random blood glucose $100 \mathrm{mg} / \mathrm{dl}$ and fasting cholesterol $380 \mathrm{mg} / \mathrm{dl}$. She died in January 1976, 5 years after the appearance of symptoms. Postmortem examination (Professor Blackwood) showed bronchopneumonia, pulmonary infarcts and calf vein thrombosis. The viscera were macro- scopically normal but no sections were available for histological examination. The brain was divided midsagittally, the left half deep frozen and subsequently samples of this were inoculated intracerebrally into laboratory animals at the National Institutes of Health, Bethesda, USA. To date no inoculated animals have developed encephalopathy but they are still under observation (Gajdusek, personal communication).

Case 4 JC. Female, born 1934. Sixth generation, IV-58 in pedigree of Rosenthal et al. ${ }^{7}$ Formerly a theatre sister. Her first symptoms appeared in spring 1974 and consisted of paraesthesiae in both thighs and difficulty in running. Subsequently her gait became more unsteady with occasional falls when turning round. In 1975 she developed intermittent diplopia on near vision, slurring of speech and clumsiness of fine movements involved in sewing and writing. She would also be woken at night with painful cramps in the limbs. On examination in March 1977 her higher functions were normal and there was minimal dysarthria. Her gait was broad-based and she showed truncal ataxia. The cranial nerves were intact and there was no nystagmus. In the limbs there was generalised hypotonia and mild cerebellar ataxia but muscle bulk and power were normal. The tendon reflexes were brisk in the arms but absent in the legs. The plantar responses were flexor and the abdominal reflexes absent. There was no sensory impairment. At this time she declined hospital admission for further investigations. She was seen again in September 1978 and admitted to hospital. She was now no longer able to walk unaided and her ataxia had increased. Her intellect was preserved but there was marked slurring dysarthria. Pursuit 
eye movements were jerky and there was first degree horizontal and vertical nystagmus. Paresis of convergence and accommodation was present. Cranial nerve functions were otherwise normal. The upper limbs were hypotonic with cerebellar ataxia but with normal muscle bulk and power; the lower limbs were mildly spastic with a pyramidal distribution of weakness. Deep tendon reflexes were increased in the arms and absent in the legs. Plantar responses were extensor. There was subjective impairment to cutaneous sensation in a stocking distribution to mid-calf level. Examination of other systems was normal. The following investigations were normal: full blood count, blood film, folate, vitamin B12, urea, electrolytes, glucose, liver function tests, urinary and plasma aminoacids, serum lipids, plasma electrophoresis, specific serology, chest and skull radiographs, ECG, EEG and visual evoked responses. The ESR was 30 $\mathrm{mm} / \mathrm{hr}$ and serum proteins showed a slight acute phase reaction. No lumbar puncture was performed. CT scan of the brain showed normal third and fourth ventricles and slight symmetrical dilatation of the lateral ventricles. Convexities and sulci appeared normal. Psychometry revealed a WAIS verbal IQ of 110 and performance IQ of 95; her memory was good. In spring of 1979 she developed impairment of recent memory, associated with a rapidly progressive deterioration in other cognitive functions. Her speech became unintelligible and she was bedridden and doubly incontinent. She developed dysphagia and drooling of saliva. On her final admission in December 1979 she showed a decorticate state. She was emaciated and anarthric, making occasional grunting noises only. Eye movements were random and roving with no real eye contact. There was a left upper motor neuron type facial weakness. Palatal and pharyngeal reflexes were exaggerated and there was a brisk jaw jerk. Brainstem reflexes were intact. Tone was increased in the limbs. The legs were fixed in a flexed, scissored posture. There was no purposeful movement in the arms which were maintained in abnormal postures. Primitive reflexes were present. No myoclonic jerks were observed. She deteriorated and died in January 1980, six years after the onset of her illness.

The clinical features of the other affected members of the family are briefly summarised in the Appendix and are included in tables 1-3.

Table 2 Presenting complaints in 16 cases

\begin{tabular}{lr}
\hline Unsteady gait & 8 \\
Disturbance of memory, cognition or affect & 4 \\
Leg weakness & 4 \\
Paraesthesiae & 4 \\
Inco-ordination arms & 3 \\
Scotomata or photopsia & 3 \\
Loss of taste and smell & 1 \\
Deafness & 1 \\
Parkinsonian features & 1 \\
\hline
\end{tabular}

\section{Material and methods}

The brain and spinal cord of case 1 were received from another hospital after fixation, the other necropsies being done at the National Hospital. A few blocks were taken from case 2 in 1973 and were intended to be for a preliminary study. However this brain is now no longer available and the survey of the neuropathology in this case is therefore very limited. The brains of cases 3 and 4 were divided mid-sagittally, one half being deep frozen from which samples were sent to Dr DC Gajdusek's laboratory at the National Institutes of Health, Bethesda, USA for inoculation into animals. After fixation blocks taken from many regions of cerebral and cerebellar hemispheres, brainstem and spinal cord were embedded in celloidin or in paraffin wax. Sections were stained with haematoxylin-eosin, haematoxylin-van Gieson, phosphotungstic acid haematoxylin, Holzer's method for astrocytes, Glees and Marsland's silver impregnation, luxol fast blue-cresyl violet, Congo red and periodic acidSchiff (PAS) methods. In cases 1-3 frozen sections were cut from a frontal block and stained for fat; for microglial cells by the Weil-Davenport method and by Cajal's gold-sublimate method for astrocytes.

\section{Neuropathology}

In all cases significant pathology was confined to the nervous system. All the brains showed a slight degree of atrophy affecting gyri of frontal, parietal and cerebellar

Table 1 Neurological systems affected in 16 cases

\begin{tabular}{|c|c|c|c|c|c|c|c|c|}
\hline & Age at onset & $\begin{array}{l}\text { Duration } \\
\text { (years) }\end{array}$ & Cerebellar & Pyramidal & $\begin{array}{l}\text { Lower } \\
\text { motoneuron }\end{array}$ & Sensory & Cranial nerves & Dementia \\
\hline $\mathbf{A M}$ & 54 & 4 & + & $\ldots$ & $\ldots$ & $\ldots$ & $\ldots$ & $\ldots$ \\
\hline CR & 66 & 3 & $?$ & $\cdots$ & $\cdots$ & + & $\cdots$ & + \\
\hline TW & 47 & 5 & + & $\ldots$ & $\ldots$ & + & + & + \\
\hline DC & 46 & 3 & + & + & $\cdots$ & $\cdots$ & $\cdots$ & + \\
\hline ES & 56 & $? *$ & $\ldots$ & + & $\cdots$ & $\cdots$ & $\cdots$ & $\cdots$ \\
\hline EU & 57 & 5 & + & + & $\ldots$ & $\ldots$ & $\cdots$ & + \\
\hline GH & 57 & 3 & + & + & $\cdots$ & + & + & + \\
\hline $\mathbf{J M}$ & 60 & 3 & $\cdots$ & + & $\cdots$ & $\cdots$ & $\cdots$ & + \\
\hline TM & 66 & 3 & $\perp$ & $\ldots$ & $\ldots$ & $\ldots$ & + & $\cdots$ \\
\hline KH & 53 & 10 & + & + & $\because$ & + & + & + \\
\hline GL & 48 & $5^{*}$ & + & $\ldots$ & + & + & + & $\cdots$ \\
\hline JW & 42 & 1 & + & + & + & $\cdots$ & + & + \\
\hline JJ & 50 & 3 & + & $\cdots$ & $\cdots$ & $\cdots$ & + & + \\
\hline BD & 38 & 7 & + & + & $\cdots$ & $\cdots$ & $\cdots$ & + \\
\hline JH & 39 & 5 & + & + & $\cdots$ & + & $\because$ & $\cdots$ \\
\hline JC & 40 & 6 & + & + & + & + & + & + \\
\hline
\end{tabular}

* Accidental death. 
Table 3 Signs in order of frequency

Unsteady gait
Slurring dysarthria
Impaired memory, cognition or affect
Spastic leg weakness
Cerebellar ataxia
Absent leg reflexes
Incontinence
Extensor plantar responses
Paraesthesiae
Nystagmus
Spastic arm weakness

cortex and there was also some dilatation of the ventricles. In all cases the major cerebral arteries were free of atheroma. There was a varying degree of neuronal loss in the cerebral cortex. This was severe and diffuse in cases 3 and 4 and milder and more patchy in cases 1 and 2 .

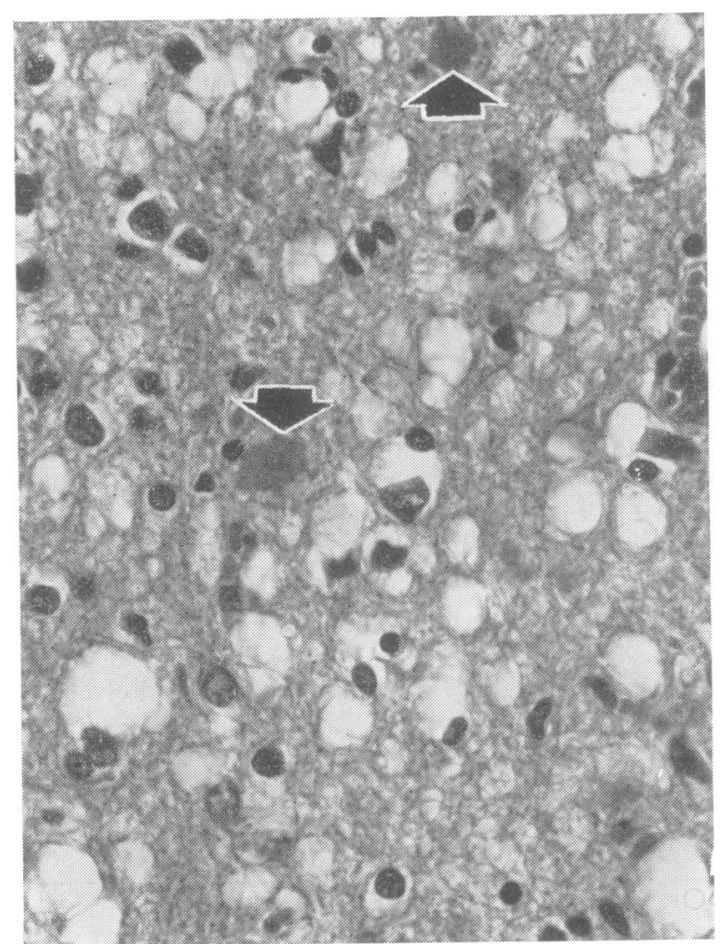

Fig 2 Case 4-Temporal cortex showing marked spongiform changes, neuronal loss and deposits of amyloid (arrows). $H$ and $E \times 375$.

Nerve cell loss in the cerebellum was a marked feature of the pathology in all four cases. Purkinje cells were absent from long stretches of cortex and empty tangles of basket cell fibres were numerous. Many of the surviving Purkinje cells showed "torpedoes". Granule cells were severely reduced in case 4 only. The cerebellar vermis showed more nerve cell loss than the hemispheres where the dorsal cortex was more severely involved than the ventral.
Numerous degenerating terminals were present close to cells of the dentate nucleus.

Status spongiosus was observed in the grey matter of all cases, varying considerably in degree and extent. In case 1 there was involvement of superior and middle frontal and superior temporal gyri, insula and parts of parietal cortex and in these areas the spongiform changes were limited to the molecular and outer nerve cell layers of the cortex. Nowhere was the full thickness of cortex affected and in many places the cortex seemed entirely spared. In case 2 a mild status spongiosus was present in the amygdaloid complex and anterior hypothalamus. Cerebral cortex and deep grey nuclei of cases 3 and 4 showed generalised status spongiosus which spared the

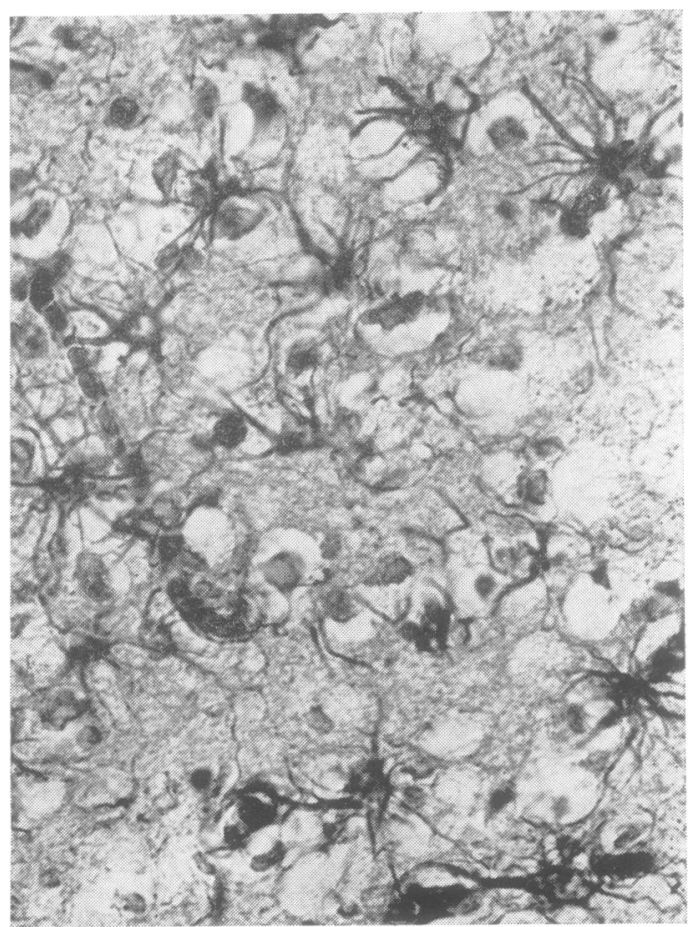

Fig 3 Case 3-Temporal cortex. Marked astrocytic hyperplasia and spongiform changes shown. Holzer's method for astrocytes $\times 400$. 


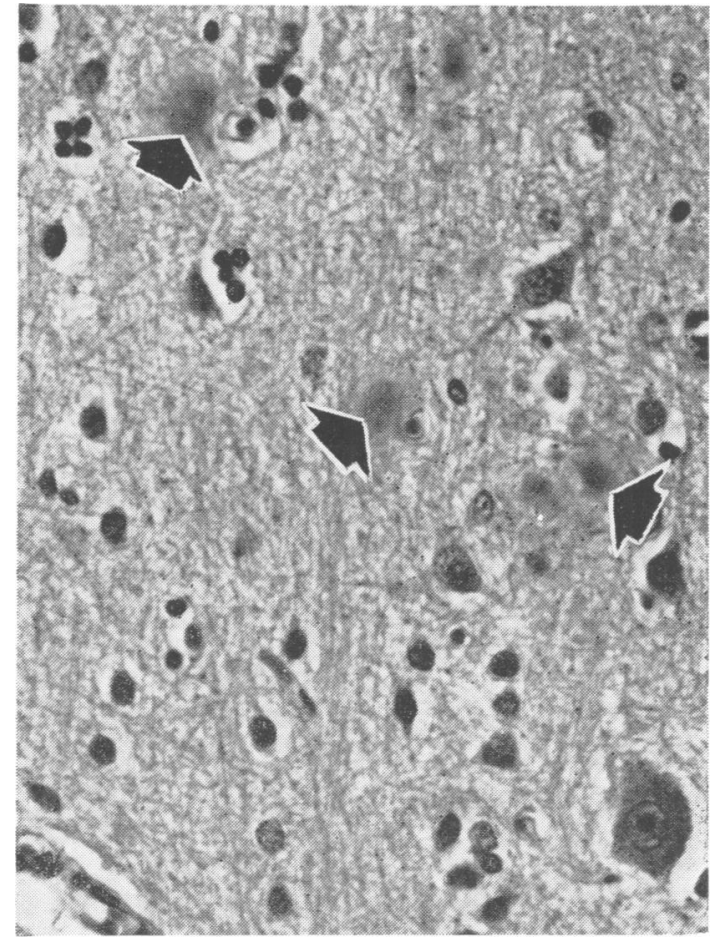

Fig 4 Case 1-Occipital cortex showing several deposits of amyloid (arrows to some) in the neuropil. There is no relationship between amyloid and iessels and no spongiform changes in this region of cortex. $H$ and $E \times 275$.

calcarine cortex in case 4 and the cerebellum. The spongiform change (fig 2) consisted of small, rounded vacuoles, of 50-100 $\mu \mathrm{m}$ in diameter, isolated or in clusters throughout the neuropil. In places the vacuoles abutted closely together but did not seem to coalesce. Vacuoles seemed to impinge on, and distort, nerve cells and in the severely spongy areas the nerve cells were shrunken.

Astrocytic hyperplasia (fig 3 ) was always a concomitant of spongiform change. Numerous astrocytes were present in the molecular layer of cerebral cortex and were particularly prominent in the deep layers of cortex close to the cortex-white matter junction. Hyperplasia of Bergmann glia and fibrous gliosis were present in many places in the molecular layer. Astrocytic fibres formed a dense feltwork in the white matter of both cerebral and cerebellar hemispheres while the ventricular ependymal lining was focally deficient and replaced by tangles of glial fibres.

Amyloid deposition (figs 4-8) was a striking feature of the pathology in all cases and was found in cerebrum (fig 4), cerebellum (fig 5) and, to a lesser extent, in the brain-stem and spinal cord. The amyloid took the form of rounded bodies ranging in size from discrete droplets of less than $5 \mu \mathrm{m}$ in diameter to large masses of more than $50 \mu \mathrm{m}$ diameter. In places the little droplets were discrete, particularly in the granular layer of the cerebellum (fig 6) but elsewhere there were larger aggregations of amyloid spheroids of varying size particularly in the molecular layer (fig 7) of both cerebral and cerebellar cortex. Some of the deposits of amyloid had the appearance of "kuru plaques" (fig 8) with a solid central core surrounded by a halo of radiating spicules of less dense material with a reticular appearance. The staining reactions were typical of amyloid being eosinophilic and hyaline with a fairly strong positive reaction with PAS, a well-marked staining with Congo red which also conferred greenish-yellow birefringence in polarised light. The distribution of amyloid was very widespread, affecting molecular layer as well as all layers of cortex and deep grey matter. Amyloid deposits were also seen in white matter but were not numerous. They were not perivascular and were not argyrophilic.

In case 3 only, amyloid infiltration of vessel walls (congophilic angiopathy) was present (fig 9) with the typical staining characteristics. Intracerebral capillaries were not affected.

Neuritic plaques of the senile (Alzheimer) type were also identified but were few in number and present mainly in the hippocampus in case 3 and scattered in the cortex in case 4 . These neuritic plaques were larger than the much more numerous amyloid deposits. Some had a

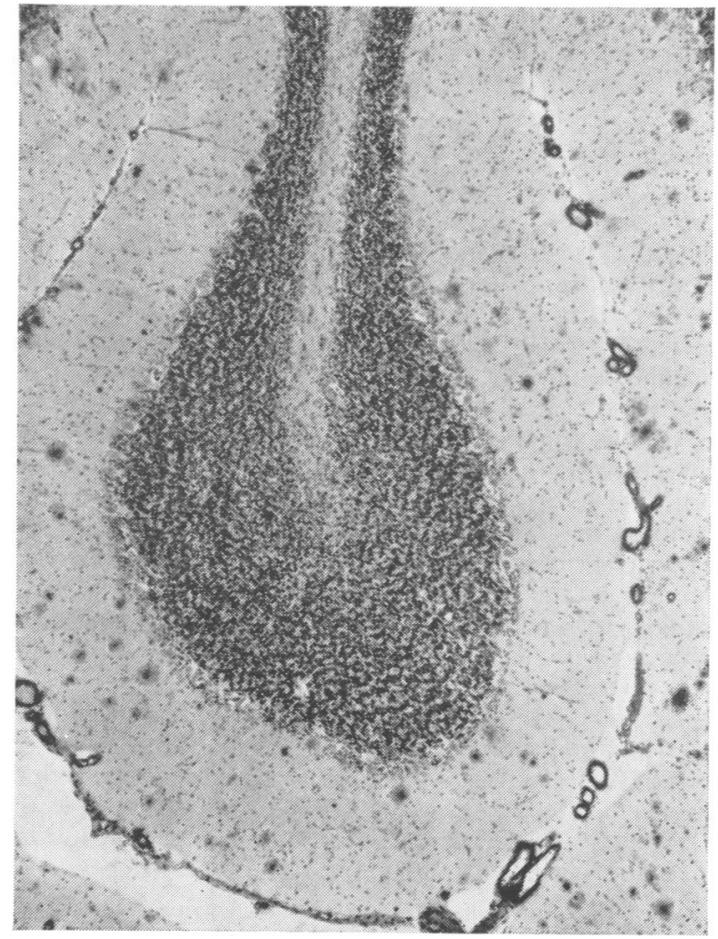

Fig 5 Case 3-Celloidin section of cerebellum. Many amyloid deposits of varying size are scattered throughout the molecular layer and the amyloid infiltration of meningeal vessels is also seen. $P A S \times 30$. 


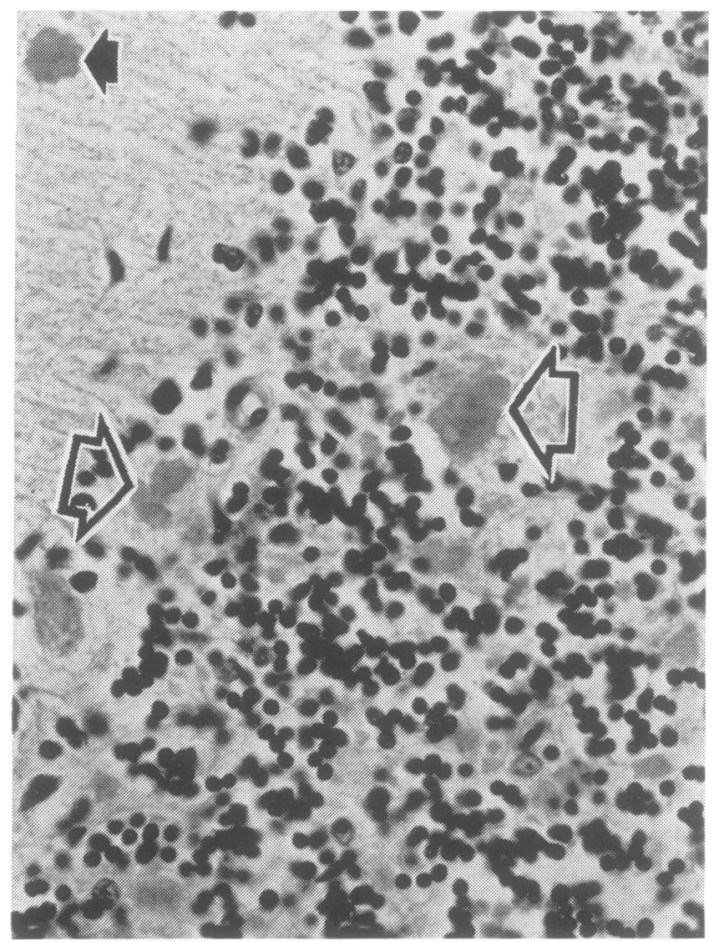

Fig 6 Case 4-Cerebellar cortex. In addition to the larger rounded masses of amyloid (arrows) there are very many small deposits between the granule cells. Purkinje cell loss is also apparent. Congo red $\times 350$.

central core of amyloid surrounded by argyrophilic bodies, astrocytes and microglial cells. In case 3 there were occasional hippocampal neurons with neurofibrillary tangles and some with granulo-vacuolar inclusions.

Venous channels were engorged with blood in case 4 (in which there had been terminal cerebral sinus thrombosis). There were also occasional perivascular cuffs of lymphocytes in the brainstem. Also in case 4 (the most extensively necropsied case) vacuolated nerve cells were present in the lumbar anterior horn and sensory root ganglia showed marked hyperplasia of satellite cells and numerous nodules of Nageotte. Several small, old ischaemic infarcts were noted in the cerebellar and occipital cortex of case 3 and throughout the white matter there was marked perivascular rarefaction, changes which could be ascribed to the amyloid vascular disease. Sections of skeletal muscles (case 4, sternomastoid, deltoid) showed atrophied muscle fibres with a fascicular distribution typical of neurogenic atrophy.

\section{Discussion}

The pathological findings of neuronal degeneration, particularly affecting Purkinje cells, astrocytic hyperplasia and status spongiosus are strongly indicative of CJD in the four cases reported here. The differences in severity and distribution of status spongiosus (taking the limited histological study of case 2 into account) could be ascribed to the known variation in the manifestations of $\mathrm{CJD}^{2}$ and we consider the changes in all these cases to be indicative of that disease. The long duration of illness and the late development of intellectual deterioration are, however, untypical of CJD in which most cases have a subacute course of less than 2 years with an earlier onset of dementia. ${ }^{3}$ However in cases of CJD in whom the main pathology is cerebellar ${ }^{8}$ dementia may develop late, or even terminally.

Amyloid plaques in $\mathrm{CJD}^{9-11}$ are closely similar to those in kuru ${ }^{12}$ and scrapie ${ }^{13}$ and are described as stellate cores. ${ }^{14}$ The finding of amyloid angiopathy, present in case 3, has not, to our knowledge, been described in cases of CJD but has been observed in presenile dementia particularly when there has been a familial pattern of inheritance or with a predominantly cerebellar involvement. ${ }^{15-18}$ Unlike other reported cases with cerebral amyloid angiopathy, neuritic argyrophilic plaques were an inconspicuous feature of the pathology of our cases

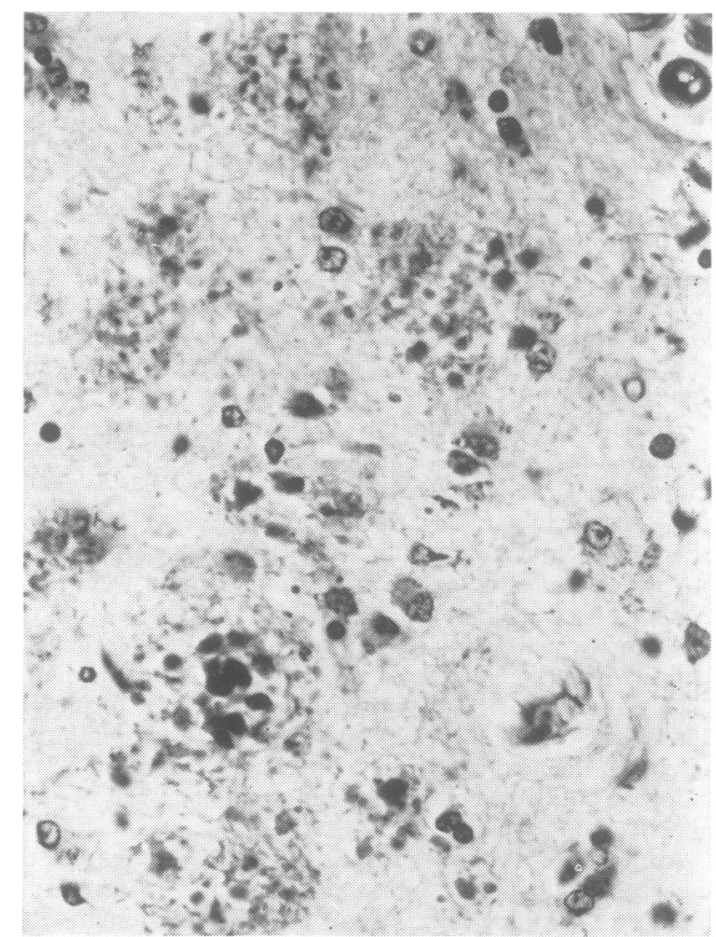

Fig 7 Case 4-Molecular layer of temporal cortex, close to the pia. Innumerable amyloid deposits are seen, some aggregated into rounded masses, and showing no association with cerebral blood vessels. PAS $\times 350$. 


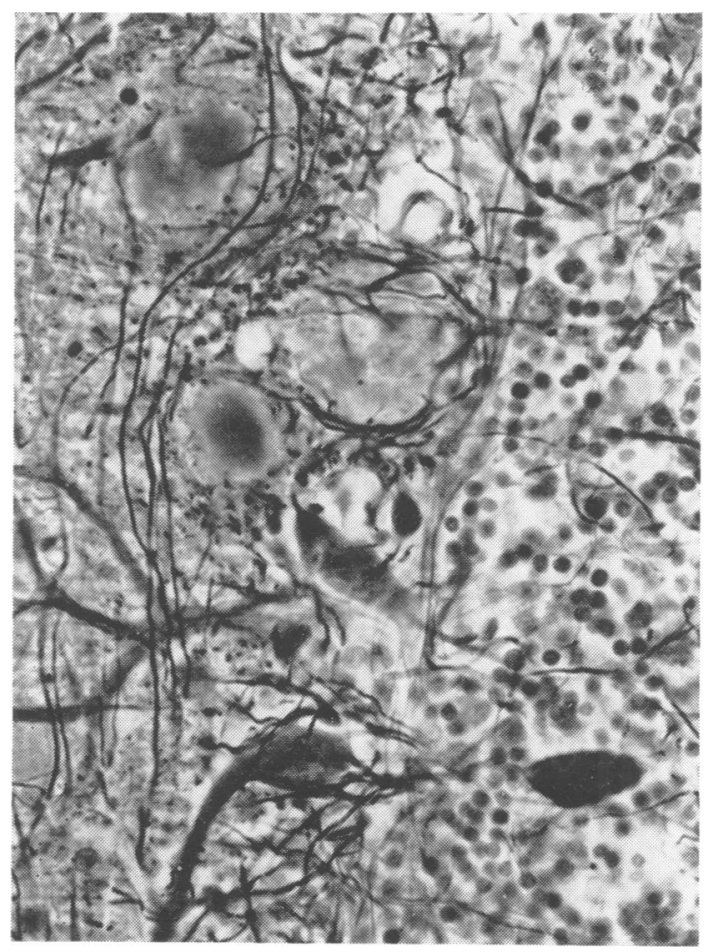

Fig 8 Case 1-Cerebellum. A torpedo on a degenerating Purkinje cell axon lies in the granule cell layer while there are several rounded deposits of amyloid resembling the so-called "kuru plaques" lying between Purkinje cells. Glees and Marsland silver impregnation $\times 350$.

while status spongiosus was prominent. No mention of spongiform change appears in the published case descriptions of amyloid angiopathy although astrocytic glial hyperplasia has been described around neuritic plaques and in the molecular layer of the cerebellum where there was also cell loss and torpedoes. ${ }^{1516}$ The familial cases reported by Gerstmann, Straussler and Scheinker ${ }^{21}$ and Boellaard and Schlote 22 were very similar to the "W" family with amyloid, spongiform changes and astrocytic hyperplasia in association with progressive cerebellar ataxia and dementia. As in the "W" family inheritance was by a dominant mode of transmission. Although mental deficiency is known in association with familial ataxia or spastic paraplegia beginning in childhood, the family reported by Akelaitis ${ }^{19}$ was an example showing severe dementia with ataxia. However the pathology in this family is uncertain.

Families with CJD have been described in many reports. ${ }^{34}$ Matthews $^{20}$ in a survey of the epidemiology of CJD in England and Wales did not describe any familial cases. We do not know whether or to what extent there was contact between the various branches of the "W" family.

We feel that the evidence presented in this paper strongly indicates that all the affected members of this family suffer from a form of CJD and a form of cerebral amyloidosis. It is not known whether the inherited disease is amyloidosis which has predisposed the CNS to superadded infection or whether the CJD virus itself has become incorporated into the genome of the host in linkage disequilibrium with the genes which predispose to amyloidosis.

We thank Mr A Beckett for the microscopic preparations and Mr JA Mills for the photographs. Some of the material was made available by Professor Blackwood and we are also grateful to Dr MFT Yealland for permission to study case 4 during her first admission.

\section{References}

${ }^{1}$ McMenemey WH. In: Greenfield's Neuropathology, 2nd ed. London: Edward Arnold, 1963:558-69.

${ }^{2}$ Corsellis JAN. Ageing and the Dementias. In: Blackwood W, Corsellis JAN, eds. Greenfield's Neuro-

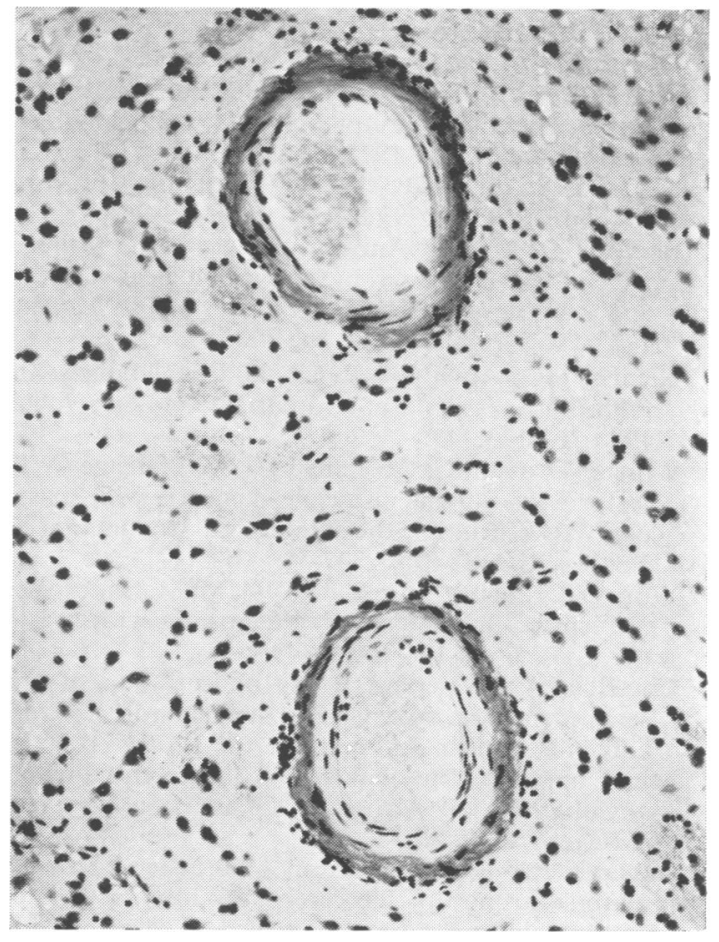

Fig 9 Case 3-Vessels in the putamen show infiltration of their walls by amyloid. Celloidin section. Congo red $\times 150$. 
pathology, 3rd ed. London: Edward Arnold, 1976: 796-848.

${ }^{3}$ Kirschbaum WR. Jakob-Creutzfeldt Disease. New York: Elsevier, 1968.

${ }^{4}$ Masters CL, Gajdusek DC, Gibbs CJ, Bernoulli C, Asher DM. Familial Creutzfeldt-Jakob disease and other familial dementias: an inquiry into possible modes of transmission of virus-induced familial diseases. In: Pruisner SB, Hadlow WJ, eds. Slow Transmissible Diseases of the Nervous System. New York: Academic Press, 1979;Vol. 1:143-93.

${ }^{5}$ Beck E, Daniel PM, Matthews WB, et al. CreutzfeldtJakob disease: the neuropathology of a transmission experiment. Brain 1969;92:699-716.

6 Cameron E, Crawford AD. A familial neurological disease complex in a Bedfordshire community. J Royal College Gen Pract 1974;24:435-6.

${ }^{7}$ Rosenthal NP, Keesey J, Crandall B, Brown WJ. Familial neurological disease associated with spongiform encephalopathy. Arch Neurol 1976;33: 252-9.

${ }^{8}$ Brownell B, Oppenheimer DR. An ataxic form of subacute presenile polioencephalopathy (Creutzfeldt-Jakob disease). J Neurol Neurosurg Psychiatry 1965;28:350-61.

${ }^{9}$ Chou SM, Martin JD. Kuru-plaques in a case of Creutzfeldt-Jakob disease. Acta Neuropath 1971 ;17: 150-5.

10 Adams JH, Beck E, Shenkin AM. Creutzfeldt-Jakob disease: further similarities with kuru. J Neurol Neurosurg Psychiatry 1974;37:195-200.

${ }^{11}$ Hayek J, Ulrich J. Kuru-plaques in Creutzfeldt-Jakob disease. Eur Neurol 1975;13:251-7.

12 Klatzo I, Gajdusek DC, Zigas V. Pathology of kuru. Lab Invest 1959;8:799-847.

13 Bruce ME, Fraser $\mathrm{H}$. Amyloid plaques in the brains of mice infected with scrapie: morphological variation and staining properties. Neuropath Appl Neurobiol 1975;1:189-202.

14 Glenner GG. Amyloid deposits and amyloidosis: the $\beta$-fibrilloses (second of two parts). New Engl J Med 1980;302:1333-43.

15 Worster-Drought C, Greenfield JG, McMenemey WH. A form of familial presenile dementia with spastic paralysis. (Including the pathological examination of a case.) Brain 1940;63:237-54.

16 Worster-Drought C, Greenfield JG, McMenemey WH. A form of familial presenile dementia with spastic paralysis. Brain 1944;67:38-43.

17 Corsellis JAN, Brierley JB. An unusual type of presenile dementia. (Atypical Alzheimer's disease with amyloid vascular change.) Brain 1954;77:571-87.

18 Mandybur TI. The incidence of cerebral amyloid angiopathy in Alzheimer's disease. Neurology (Minneap) 1975;25:120-6.

19 Akelaitis AJ. Hereditary form of primary parenchymatous atrophy of the cerebellar cortex associated with mental deterioration. Amer J Psychiatry 1938; 94:1115-40.

${ }^{20}$ Matthews WB. Epidemiology of Creutzfeldt-Jakob disease in England and Wales. $J$ Neurol Neurosurg Psychiatry 1975;38:210-3.
${ }^{21}$ Gerstmann J, Sträussler E, Scheinker J. Uber eine eigenartige hereditazfamiliare Erkrankung des Zentralnervensystems. Zeitschrift für Neurol 1936;154: 736-62.

${ }^{22}$ Boellaard JW, Schlote W. Subakute spongiforme Encephalopathie mit $\mathrm{m}$ ltiformer Plaquebildung. Acta Neuropath 1980;49:2 5-12.

\section{Appendix}

No details are known of the illnesses in the affected members of generations I and II. The histories of GH, $\mathrm{TM}, \mathrm{KH}, \mathrm{BD}$, and $\mathrm{MJ}$ have been compiled from their case notes. The others are from Rosenthal et al. ${ }^{7}$

\section{Generation III}

$M W$ (female) A daughter of this lady stated that her mother and her identical twin both died aged 55 after a similar disabling illness, the cause of death being stated as disseminated sclerosis.

\section{Generation IV}

AM(female 1883-1941) Mother of KH and grandmother of case 4. Gradual development of slurred speech, decreasing use of legs and progressive incapacitation without dementia or memory loss. Her handwriting became small.

CR (female 1891-1960) At age 66 developed pain in legs that caused difficulty in walking. Speech became incoherent and she became incontinent.

TW (male 1894-1946) The father of JW. Illness began 5 years before death, beginning with difficulties with balance. Became completely disabled within 2 years with slurred speech, difficulty with swallowing, "black balls" in his vision, numbness in extremities and impaired hearing. Family deny any evidence of dementia, memory loss or confusion until 3 weeks before death when he lapsed into coma.

DC (female 1894-1943) Developed headaches and menorrhagia at age 46 accompanied by inability to knit and difficulty with walking. During severe headaches her memory and orientation would deteriorate. Became bedridden.

ES (female 1903-1959) Died as a result of a fall down stairs which was attributed to weakness of her legs similar to that of other affected members of the family.

\section{Generation V}

EU (female 1914-1976) Case 3 of this paper.

GH (female 1909-1968) Illness began at age 57 with difficulty in use of legs, tingling sensation in the face and a black spot in the left visual field. Neurological examination May 1965 showed an inexpressive face, cogwheel rigidity of both arms, weakness of hip flexion and bilateral extensor plantar responses. By February 1967 there was marked unsteadiness, nystagmus on lateral gaze, gross dysarthria and truncal weakness. Gait was slow and shuffling with difficulty in turning round, marked weakness of hip and knee movements and ataxia of legs. Tendon reflexes brisk, in upper limbs but absent in lower limb with bilateral extensor plantars. She was well orientated and able to concentrate on questions but 
was severely depressed and apathetic. By 1968 she was able to walk only with support and in that year was admitted to a mental hospital where she died some months later.

$J M$ (male 1912-1975) Case 1 of this paper.

TM (male 1903-1972) Illness began May 1969 with a brief episode of staggering gait and difficulty climbing stairs. In September 1969 noticed flashes of light in left temporal field. On examination his gait was ataxic, facies were Parkinsonian and there was bilateral anosmia and nerve deafness, more severe on left. Tendon reflexes were absent at the knees and reduced at ankles. Ataxia and dysarthria were observed in February 1971 and these progressed so that by December 1971 he was confined to a wheelchair. He died in May 1972. There was no record of dementia developing in this case.

KH (female 1908-1972) Mother of case 4. Illness began 1961 with weakness and dragging of left leg and paraesthesiae in that leg and hands. Examination showed mild left hemiparesis with extensor plantar response with sensation and cranial nerve function normal. Trigeminal neuralgia in 1966. By December 1969 both legs were weak and ataxic, gait was spastic and she took very small steps. Vibration sense absent below knees but all sensory modalities normal. In August 1970 examination showed marked slurring dysarthria, nystagmus on lateral gaze, paralysis of upward gaze and paresis of convergence. The left arm had become spastic, joint position sense in feet and sensation to pinprick (between $\mathrm{T} 7$ and L1) were impaired. In October 1971 she was admitted to hospital because of urinary infection, and by that time was quadriplegic and doubly incontinent. There is no information about her mental state throughout the course of the illness but she was transferred to a mental hospital in February 1972 and died there soon after. No necropsy was performed.

GL (female 1916-1969) In 1964 she developed difficulties with balance and a tendency to fall. By 1966 her handwriting began to deteriorate and later there was intermittent diplopia. Examination in 1967 showed ataxia of legs, absent tendon reflexes throughout and impairment of vibration and position sense in the legs. Pupils were small and irregular with no reaction to light or accommodation. Later she developed ataxia of arms and dysphagia and other neurological deficits progressed. EMG showed widespread evidence of lower motor neuron disease with normal nerve conduction velocities. In September 1969 she died 3 weeks after a fall in which she struck her head. JW (male 1930-1973) This case was reported by Rosenthal et al. ${ }^{7}$ He was well until early 1972 when he developed visual disturbances, disturbances of balance, delusions and confusion. By October 1972, he was generally unresponsive, with no voluntary eye movements, frequent jerking of all limbs and bilateral extensor plantar responses. EMG and muscle biopsy showed evidence of lower motor neuron disease. Treatment with amantadine hydrochloride (200-600 mg daily) for six weeks was not beneficial and he died 13 months after the onset of his illness. Postmortem examination showed the characteristic cerebral spongiform changes of CJD and inoculation of cerebral tissue into nonhuman primates has transmitted an encephalopathy.

$J J$ (female 1920-1973) Case 2 of this paper.

$B D$ (female 1922-1968) Admitted to a mental hospital in 1962 because of depression and forgetfulness. She could no longer work with figures and could no longer knit. Examination showed disorientation, gross memory loss and inability to concentrate for more than a few minutes. Speech was normal with mild dyspraxia. In January 1965 there were no neurological deficits but psychometry showed severe, catastrophic intellectual deterioration. By May 1965 she was noted to stand and walk unsteadily, on a broad base, with a tendency to lean to the left. By late 1965 she was unable to feed herself and was doubly incontinent. The dementia progressed slowly and in May 1967 she was noted to make few voluntary movements and was unable to sit unsupported. Her left side was paralysed by December 1967 and she died of pneumonia in January 1968.

\section{Generation VI}

JH (male 1931-1975) Brother of case 4. Developed unsteadiness of gait in 1970, progressive dysarthria from 1971 and dysphagia from 1972. Paraesthesiae of hands and feet and distortion of his handwriting then developed. Examination in 1973 showed no intellectual impairment, speech was dysarthric and gait broad-based. Ocular movements were full and pupillary responses normal but there was nystagmus in all directions of gaze. Vibration and position sense were impaired in feet and there was weakness of knee and hip flexors. All limbs showed ataxia, tendon reflexes were present but plantar responses were extensor bilaterally. He died after a 5 year illness without dementia (no necropsy).

JC (female 1934-1980) Case 4 of this paper.

MJ (female, born 1952, daughter of JJ) She did not walk until 3 years old, had many attacks of loss of consciousness. There was evidence of marked mental retardation and there have been many admissions to a mental hospital for multiple overdoses, hysterical fits and outbursts of aggression. She is considered to have a behavioural disorder in association with epilepsy and subnormality. 